\section{Adaptação transcultural parcial da escala Aberrant Behavior Checklist (ABC), para avaliar eficácia de tratamento em pacientes com retardo mental}

\author{
Partial cross-cultural adaptation of the Aberrant \\ Behavior Checklist (ABC) scale for analysis of \\ patients with mental retardation
}

\author{
1 Escola Bahiana de Medicina \\ e Saúde Pública, Salvador \\ Brasil. \\ 2 Universidade Federal da \\ Bahia, Salvador, Brasil. \\ ${ }^{3}$ Hospital Santo Antônio, \\ Salvador, Brasil. \\ 4 Faculdade Ruy Barbosa, \\ Salvador, Brasil. \\ 5 Secretaria Estadual de \\ Justiça, Cidadania e Direitos \\ Humanos do Estado da \\ Bahia, Salvador, Brasil. \\ ${ }_{6}^{6}$ Faculdade de Tecnologia e \\ Ciências, Salvador, Brasil. \\ 7 Universidade Federal do \\ Rio de Janeiro, Rio de Janeiro, \\ Brasil. \\ Correspondência \\ M. F. Losapio \\ Escola Bahiana de Medicina e \\ Saúde Pública. \\ Rua Frei Henrique 8, Salvador \\ BA 40050-420, Brasil. \\ mfl_ssa@hotmail.com
}

\section{Abstract}

The aim of the ABC (Aberrant Behavior Checklist) is to evaluate the treatment response for aberrant behavior in patients with mental retardation. The aim of this study was to describe the partial cross-cultural adaptation of the ABC scale to Brazilian Portuguese. The process included conceptual and item equivalence, two translations ( $T 1$, $T 2)$ and their back-translations (R1, R2), evaluation of referential and general equivalence, expert evaluations, a pre-test, and elaboration of the final version. Conceptual and item equivalences of the $A B C$ were considered pertinent to Brazilian culture. Semantic equivalence showed good correspondence between R1 items and ABC. Reasonable correspondence was obtained between $A B C$ items and R2. All of the professors understood 94.8\% of the items in the scale, while relatives understood 87.9\%. The Brazilian Portuguese version of the $A B C$ scale thus is available for use, with the appropriate conceptual, item, and semantic equivalence.

Translating; Mental Retardation; Treatment Outcomes; Questionnaires

\author{
Mirella Fiuza Losapio 1 \\ Lis Gomes Silva 2 \\ Milena Pereira Pondé 1 \\ Camila Marinho Novaes 3 \\ Darci Neves dos Santos 2 \\ Nayara Argollo 4 \\ Ivete Maria Santos Oliveira 5,6 \\ Heloisa Helena Alves Brasil 7
}

\section{Introdução}

O retardo mental é um dos transtornos psiquiátricos mais comuns em crianças. Apresenta prevalência de $2 \%$ a $3 \%$ na população jovem 1 . Entre crianças autistas a prevalência de retardo mental é ainda maior 2,3. O diagnóstico de retardo mental requer a presença de três critérios: início do quadro clínico antes de 18 anos de idade; função intelectual significativamente abaixo da média, demonstrada por um quociente de inteligência (QI) igual ou menor que 70; e deficiência nas habilidades adaptativas em pelo menos duas das seguintes áreas: comunicação, autocuidados, habilidades sociais e interpessoais, auto-orientação, rendimento escolar, trabalho, lazer, saúde e segurança 4 .

Pesquisas demonstram que a prevalência de transtornos comportamentais é significativamente maior na população com retardo mental em comparação com a população geral 5 . Cerca de 70 a $85 \%$ dos pacientes com transtorno de desenvolvimento apresentam alterações comportamentais que não são tratadas ou sequer diagnosticadas. Muitas destas condições podem acarretar delírios e quadro psicótico 6,7. Apenas cerca de $5 \%$ a $10 \%$ dos portadores de retardo tornam-se adultos independentes, $25 \%$ têm alguma melhora, mas ainda necessitam de supervisão, enquanto que o restante continua incapacitado, requerendo institucionalização para melhores cuidados, redundando em alto custo familiar 
e comunitário ${ }^{8}$. Sua causa permanece desconhecida, variando desde infecção, genética, teratogenicidade, neurotransmissão e receptores anormais. O principal tratamento consiste em medidas socioeducativas. A farmacoterapia pode ser necessária para reduzir sintomas e comportamentos específicos em pacientes com transtornos mais graves, para os quais as abordagens psicoeducativas habituais são ineficientes 9 .

Existem diversas escalas para avaliação de habilidades cognitivas, dentre elas as mais utilizadas são as Escalas Wechsler de Inteligência, com suas variantes de acordo com a idade do indivíduo avaliado. Suas versões mais atualizadas são a Wechsler-Bellevue Intelligence Scale - Form III (WISC-III) 10 e a Wechsler Adult Intelligence Scale - Form III (WAIS-III) ${ }^{11}$; tendo sido a primeira desenvolvida para crianças em idade escolar e a segunda para adultos. Ambas as escalas já foram traduzidas e validadas no Brasil. Outros instrumentos já validados no Brasil são a Bateria Woodcock-Johnson III (WJ-III) 12, os testes das Matrizes Progressivas de Raven 13, o G-36 14 e Desenho da Figura Humana (DFH-III) 15.

Um instrumento de avaliação que mensure os sintomas comportamentais em pacientes com retardo mental é importante para que se possa avaliar a evolução das medidas terapêuticas utilizadas. Dentre todas as escalas já publicadas na literatura nacional e internacional, a Aberrant Behavior Checklist (ABC) é a única que tem o objetivo de avaliar a resposta aos tratamentos, em pacientes com retardo mental.

A escala ABC foi desenvolvida em 1985, na Nova Zelândia, com o objetivo de avaliar a resposta ao tratamento medicamentoso, ou a outras intervenções, em transtornos comportamentais em portadores de retardo mental moderado a profundo 16. A escala foi desenvolvida a partir de um pool de itens descrevendo comportamento alterado, aplicado a um grupo de 418 indivíduos (estágio 1), composto por adolescentes e adultos jovens institucionalizados. A lista foi submetida a uma análise fatorial e itens redundantes ou raramente endossados foram retirados, compondo-se uma versão intermediária. Esta foi posteriormente aplicada a um grupo similar de 509 indivíduos (estágio 2), para um procedimento de validação cruzada 16 (aplicação de instrumentos em duas populações diferentes a fim de comparar os resultados).

O estudo original foi constituído, predominantemente, por indivíduos do sexo masculino (60\%); com idade variando de 21-38 anos no estágio 1 e 16-31 anos no estágio 2; portadores de retardo moderado (41\%), grave (45\%) e profundo (15\%). Quanto à condição médica subjacente, houve predominância de epilepsia em ambos os estágios e, em segundo lugar, paralisia cerebral, sendo verificados ainda, em percentuais menores, surdez, paralisias não especificadas e psicose. A maioria dos pacientes eram usuários de medicamentos psicotrópicos, sendo mais frequente o uso de neuroléptico e anticonvulsivantes. Os avaliadores foram, na imensa maioria, enfermeiros das instituições participantes do estudo 16.

A escala ABC apresenta 58 itens divididos em 5 subescalas: I - irritabilidade, agitação e choro (15 itens); II - letargia e esquiva social (16 itens); III - comportamento estereotipado (7 itens); IV - hiperatividade (16 itens); V - fala inapropriada (4 itens) 17. Foram realizados vários estudos psicométricos da $\mathrm{ABC}$, que evidenciaram: reprodutibilidade interavaliador moderada 17,18 , reprodutibilidade teste-reteste alta 17,19 e a validade de critério demonstrou-se adequada a muito boa entre os estudos 18,20. A validade de fatores também foi investigada em populações na Inglaterra 21 e nos Estados Unidos 22,23. A estrutura fatorial, no geral, demonstrou ser robusta, embora tenha se verificado tendência para alguns itens dos fatores irritabilidade (I) e hiperatividade (IV) serem computados em fatores alternativos nos três estudos. Além disso, apesar de Fala Inapropriada ter emergido como fator separado no estudo de Newton \& Sturmey 21, quatro itens originalmente agrupados no fator irritabilidade (I), também foram computados em fala inapropriada (V) 24 .

Houve três estudos envolvendo crianças com retardo mental, sendo dois deles realizados na comunidade, com pacientes não institucionalizados 19,20,24. No estudo de Freund \& Reiss 19, 110 crianças, adolescentes e adultos jovens portadores de retardo, acompanhados em um serviço ambulatorial, foram avaliados por pais e professores. Quatro itens foram excluídos da análise fatorial das escalas aplicadas pelo grupo de pais, devido ao endossamento infrequente dos itens. A análise fatorial em cinco fatores resultou em estrutura similar ao do estudo original. A estrutura fatorial das avaliações dos pais demonstrou maior similaridade com o original do que a dos professores.

Mashburn \& Aman 24 realizaram estudo com 666 crianças e adolescentes que frequentavam classes de educação especial, com idades entre 6 a 21 anos (média de 12,5). Utilizaram uma versão reformulada da escala, a ser preenchida por professores, em que trazia instruções modificadas para que deixassem de existir referências restritas ao contexto de enfermaria. Neste estudo, obeteve-se uma solução de quatro fatores. Foi demonstrada alta congruência entre os quatro fatores e seus correspondentes na escala original. 
A escala é acompanhada por um cabeçalho que deve ser preenchido com as seguintes informações: características demográficas (idade, sexo, grau de retardo), qualquer diagnóstico médico ou psiquiátrico, uma lista das medicações em uso. $\mathrm{O}$ instrumento deve ter cada um dos seus itens pontuados entre 0 e 3 . Em seguida há uma folha de respostas na qual a pontuação deve ser descrita nas subescalas, de modo a acompanhar a evolução de determinado paciente em todos os cinco fatores avaliados no decorrer do tratamento.

Para que estudos clínicos ou epidemiológicos tornem-se válidos é necessário que as informações desejadas sejam colhidas através dos instrumentos padronizados. Quando o instrumento de avaliação não foi desenvolvido na cultura onde ele será utilizado, seu uso requererá um processo de tradução e adaptação abrangentes, objetivando uma equivalência cultural que não é alcançável apenas por técnicas de tradução e retradução (técnica que consiste em traduzir um documento previamente traduzido, de volta ao seu idioma original. Essa técnica permite melhorar a qualidade da tradução realizada) 25 . Fatores linguísticos e socioculturais múltiplos devem ser considerados na determinação de equivalência transcultural 26. Uma adaptação transcultural envolve tradução, ajustes culturais de palavras para o idioma e contexto ao qual está sendo traduzida, possibilitando uma melhor captura do sentido pretendido 27 .

Na literatura há diversos modelos de abordagens teóricas e propostas processuais 26 . São alguns exemplos: (1) tradução palavra por palavra, (2) tradução literal, (3) tradução exata, respeitando as normas gramaticais do texto original, (4) tradução semântica, que se preocupa mais com as características estéticas do texto, (5) tradução com adaptação transcultural, (6) tradução livre, (7) tradução idiomática, que preserva a mensagem original mas pode usar palavras mais coloquiais e (8) tradução comunicativa, que tenta manter o significado original, desde que nem a essência do texto nem a língua deixem de priorizadas ${ }^{28}$.Todavia, não há consenso quanto às estratégias de operacionalização 26 . Dessa forma, optou-se por um dos modelos possíveis, o de Herdman et al. 25 sistematizado por Reichenheim \& Moraes 27, por valorizar a adaptação transcultural, seguindo as etapas de equivalências conceitual e de itens, semântica, operacional e de mensuração.

O presente estudo tem como objetivo descrever o processo parcial de adaptação transcultural da escala $\mathrm{ABC}$ para o Brasil, envolvendo as etapas de equivalência conceitual e de itens e da equivalência semântica, de modo a torná-la disponível para a investigação quanto às propriedades psicométricas e uso futuro no contexto de ensaios clínicos.

\section{Metódos}

\section{Avaliação da equivalência conceitual e de itens}

A avaliação da equivalência conceitual consiste na investigação do instrumento de interesse e dos pesos dados aos seus domínios constituintes no país de origem e na cultura para qual pretende ser adaptado. A equivalência de itens avalia se os itens utilizados para a captação dos domínios contemplados no instrumento original são igualmente pertinentes na cultura da população-alvo, para a qual serão traduzidos 27 .

A equivalência conceitual foi avaliada pela autora M.P.P., que é psiquiatra, e endossada por um grupo de especialistas, composto por três psiquiatras e uma neuropediatra. $\mathrm{O}$ grupo avaliou também a equivalência de itens, através de um formulário da $\mathrm{ABC}$ com uma opção de resposta, enviado por email, que trazia, ao final de cada item, o enunciado "Em relação ao que o fator mede, o item:", e em seguida, as alternativas "Não corresponde"; "Tem sentido aproximado"; "Corresponde".

\section{Avaliação da equivalência semântica}

De acordo com Reichenheim \& Moraes 27 (p. 668), " a equivalência semântica envolve a capacidade de transferência de sentido dos conceitos contidos no instrumento original para a versão, propiciando um efeito nos respondentes semelhante nas duas culturas". Neste trabalho, adotou-se a sistematização utilizada em outro estudo publicado pelos referidos autores 29 , que dividia a avaliação da equivalência semântica em cinco etapas.

Na primeira etapa, foram feitas duas traduções independentes da versão original, do inglês para o português (T1 e T2), por médicas brasileiras fluentes em inglês, sendo uma delas psiquiatra infantil. Posteriormente, T1 e T2 foram enviadas a duas tradutoras profissionais, que tinham o inglês como idioma original, independentes e cegas em relação às primeiras profissionais, para que realizassem suas respectivas retraduções $\mathrm{R} 1$ e R2 (técnica que consiste em traduzir um documento previamente traduzido, de volta ao seu idioma original).

A terceira etapa consistiu na avaliação das equivalências referencial e geral. A primeira se destina à verificação da correspondência literal entre os itens do instrumento original e da versão 
traduzida. Já a avaliação da equivalência geral, consiste na apreciação do impacto cultural da palavra, da reação emocional que ela provoca nos respondentes. Portanto, a equivalacência referencial avalia o significado denotativo da palavra, enquanto que a equivalência geral avalia o seu significado conotativo 27 .

Nesta etapa, optou-se, mais uma vez, pela sistematatização utilizada em estudo de Reichenheim et al. 29. Foi realizada por uma quinta avaliadora, independente, atuante na área de saúde, proficiente em inglês, cega em relação às tradutoras e retradutoras. A apreciação da equivalência referencial foi feita através de escalas analógicas visuais 30 , com escores variando de 0 a $100 \%$, estimando, de forma percentual, o grau de semelhança literal entre o original e as retraduções. Para a avaliação da equivalência geral, utilizou-se uma classificação qualitativa, em que os itens das retraduções poderiam ser qualificados em: inalterado, pouco alterado, muito alterado ou completamente alterado; em relação ao significado conotativo da versão original.

A quarta etapa da avaliação semântica envolveu o grupo de especialistas da avaliação conceitual e de itens, acrescido do avaliador independente da terceira etapa, para identificação e manejo dos problemas encontrados nas etapas anteriores. Foi então elaborada uma versãosíntese, incorporando itens oriundos de $\mathrm{T} 1$ ou de T2, bem como itens híbridos ou totalmente reformulados, buscando-se a inclusão dos que melhor atendessem aos critérios de equivalência exigidos.

A quinta e última etapa, o pré-teste da versão síntese, foi realizada por duas concluintes do curso de medicina previamente treinadas. Teve como objetivo avaliar a aceitabilidade e compreensão da escala por parte da população-alvo, garantindo-se a participação anônima e voluntária dos indivíduos da amostra. Foram entrevistados 20 professores de três escolas especializadas de Salvador, Bahia: dez profissionais do Instituto Pestalozzi, sete do Centro Educacional da APAE (Associação dos Pais e Amigos dos Excepcionais) e três da AAMA (Associação dos Amigos dos Autistas). O recrutamento de voluntários se deu mediante contato prévio com coordenadores pedagógicos das referidas instituições e solicitação verbal ou por ofício, para a realização do pré-teste da versão em português realizada pelo grupo de pesquisa. As entrevistas foram feitas de acordo com a disponibilidade e aceitação dos professores em participar, durante intervalos entre aulas.

Respeitando o desenho da escala original, que foi concebida para ser preenchida por pessoas próximas ao paciente em avaliação, que podem ser professores, pais ou cuidadores, entrevistaram-se também 20 familiares de crianças e adolescentes portadores de retardo, matriculados na AAMA, também mediante solicitação à Coordenação para utilização do espaço e aceitação, por parte dos familiares, em serem voluntários. Tratou-se, portanto, de uma amostra de conveniência, composta por 40 indivíduos, divididos de forma paritária entre professores e familiares de crianças e adolescentes com retardo, selecionados de acordo com a sua disponibilidade em conceder entrevista. Como informação pessoal exigia-se apenas a escolaridade, sem restrição quanto ao grau, desde que o entrevistado fosse alfabetizado.

As entrevistas foram individuais e consistiram na leitura em voz alta dos 58 itens da escala, pedindo-se a cada voluntário que acompanhasse com uma cópia da versão-síntese em mãos. Os entrevistados eram solicitados a parafrasear cada item ou dar exemplos de situações que ilustrassem o seu conteúdo. Adicionalmente, pedia-se que também usassem o seu exemplar da escala para sublinhar palavras ou frases de vocabulário desconhecido por eles. A compreensão era julgada pelos avaliadores (duas autoras do estudo) de acordo com a pertinência das reformulações e exemplos dados, no momento da entrevista, e posteriormente, a partir da verificação dos termos sublinhados.

Os itens não compreendidos por $15 \%$ ou mais da amostra, valor fixado arbitrariamente para direcionamento dos esforços de análise, voltariam a ser avaliados pelas autoras do estudo. Estas discutiriam então a necessidade de alterar os itens assinalados, bem como a possibilidade de fazê-lo sem prejuízo da equivalência conceitual ou das equivalências referencial e geral.

\section{Resultados}

\section{Equivalência conceitual e de itens}

O grupo de especialistas concluiu que os padrões de comportamento alterado, descritos na $\mathrm{ABC}$, são pertinentes ao contexto dos portadores de retardo, na nossa cultura. No entanto, o grupo identificou, com frequência, uma abundância de itens com o mesmo sentido, no estudo original, o que poderia tornar a escala cansativa para a população-alvo, dificultando a sua aderência e correto preenchimento, e contraproducente no contexto dos ensaios clínicos. Os itens mais problemáticos nesse sentido foram: 1, 15, 38, 9 e 54; o grupo 3, 5, 16, 30 e 42; o grupo 6, 11, 27, 35, 45 e 49 e as questões 8, 19 e 41 e o par 2 e 50 . Ainda assim, concluiu-se que a sua tradução seria válida, 
ainda que para ser modificada futuramente, com o respaldo de estudos psicométricos (Tabela 1).

\section{Equivalência semântica}

Houve uma boa equivalência entre os itens de $\mathrm{R} 1$ e do instrumento original, e equivalência razoável entre estes e R2 (Tabela 1). Dessa forma, tendeu-se a incorporar mais itens de $\mathrm{T} 1$ no instrumento traduzido. Dezessete itens foram integralmente originados de T1 $(\approx 29 \%), 12$ de T2 $(\approx$ $21 \%)$ e 29 (50\%) foram modificações a partir de T1 e, menos frequentemente, de T2 (Tabela 2).

Observou-se, durante o pré-teste, aceitação razoável da escala por parte dos professores e familiares. A principal queixa era com relação à repetitividade dos itens, o que acabava tornando a entrevista cansativa, segundo relatos.

A amostra de professores de escolas especializadas foi composta por $10 \%(n=2)$ de profissionais com nível superior incompleto, $65 \%(\mathrm{n}=13)$ com nível superior completo e $25 \%(n=5)$ com pós-graduação em andamento.

A compreensão foi universal, na amostra de professores, para 55 itens (94,8\% da escala). Os itens que geraram maior frequência de dúvidas foram: o 12, em que o termo "absorto" era desconhecido para $25 \%$ da amostra $(n=5)$; o item 17, no qual o termo "excêntrico" não foi compreendido por $30 \%(\mathrm{n}=7)$; e o item 45 , em que o termo "extremidades", não foi entendido por $15 \%$ da amostra $(n=3)$, dentro do contexto em que aparecia (Tabela 3). Portanto, apenas 3 itens da escala não foram compreendidos por $90 \%$ ou mais da amostra, que foram os itens 12, 17 e 45, com os repectivos percentuais de compreensão: $75 \%, 70 \%$ e $85 \%$. A parcela da amostra que compreendeu a versão preliminar da escala de forma integral foi composta, em sua maioria, por profissionais com nível superior completo (90\%), incluindo-se todos os profissionais com pós-graduação em andamento.

Com relação à escolaridade, a amostra de familiares de portadores de retardo foi mais heterogênea e apresentou maior frequência de graus de escolaridade mais baixos. Foi composta por $15 \%$ ( $\mathrm{n}=3$ ) de indivíduos com ensino fundamental incompleto, $20 \%(n=4)$ com ensino fundamental completo, $10 \%(n=2)$ com ensino médio incompleto, $30 \%(n=6)$ com ensino médio completo, $5 \%(n=1)$ com ensino superior incompleto e $20 \%$ $(\mathrm{n}=4)$ com ensino superior completo. Quanto à compreensão dos itens da escala, na amostra dos familiares, ela foi universal para $87,9 \%$ dos itens $(\mathrm{n}=51)$. Os itens que geraram dúvidas quanto ao vocabulário empregado foram os itens 11, particularmante a expressão "comportamentos estereotipados”, não compreendida por $35 \%(\mathrm{n}=7)$; o item 12, novamente o termo "absorto", desconhecido por $30 \%(\mathrm{n}=6)$; o item 17 , tanto o termo "excêntrico", quanto "bizarro" geraram dúvida em 20\% ( $n=4)$ dos indivíduos; o item 45, com o termo "extremidades", desconhecido por $25 \%$ e o item 56, em que o termo "deliberadamente" não foi entendido por $15 \%(n=3)$ (Tabela 3). O item 37 , em sua totalidade, "indiferente às atividades estruturadas (não reage)", não foi compreendido por $35 \%(n=7)$. Durante a entrevista, teve-se a impressão de que os indivíduos não compreendiam bem o sentido da palavra "indiferente" na frase ou não conseguiam recordar o ambiente de aplicação da escala, que pode ser não só a casa como a sala de aula, parecendo necessitarem de exemplos para "atividades estruturadas". O item 20, "expressão facial imóvel, fixa, falta de resposta emocional", não foi entendido por $15 \%(\mathrm{n}=3)$. Aparentemente, a amostra de familiares estava menos familiarizada com os termos empregados neste item. Portanto, sete itens da escala não foram compreendidos por $90 \%$ ou mais da amostra, que foram os itens 11, 12, 17, 37, 45, 56 e 20, com os respectivos percentuais de compreensão: $65 \%, 70 \%, 80 \%, 65 \%, 75 \%, 85 \%$ e $85 \%$.

O item 12 deixou de conter o termo "absorto", tornando-se "preocupado(a), distraído(a), fixa o olhar no vazio", sem prejuízo em relação à versão original ("preoccupied; stares into space”). A palavra "absorto" havia sido incorporada pelos autores, junto ao avaliador das equivalências, como adjetivo para a expressão "fixa o olhar no vazio", junto ao sinônimo "distraído", mas acabou por confundir grande parte da amostra e por isso optou-se por retirá-la do item.

O item 17, "excêntrico, comportamento bizarro”, foi modificado, conforme versão proposta em T2, para "estranho, comportamento esquisito", vocabulário mais coloquial, portanto mais acessível e sem prejuízo das equivalências referencial ("odd, bizarre in behavior") e geral, por apresentar termos sinônimos.

Para o item 37, “indiferente às atividades estruturadas (não reage)", optou-se pela substituição por versão proposta em T1, "não acompanha as atividades estruturadas (não reage)", que embora represente uma distorção da literalidade em relação à versão original, "unresponsive to structured activities (does not react)", não compromete o significado geral e, supõe-se, mantém uma conotação condizente com o que o fator original avalia: letargia, isolamento social.

No item 45, o termo "extremidades" foi substituído por "mãos ou pés", uma vez que a versão original, "waves or shakes the extremities repeatedly”, apresenta um jargão médico, desconhecido tanto pela amostra de professores como de familiares. 
Tabela 1

Comparação entre o original em inglês e as "retrotraduções" do instrumento Aberrant Behavior Checklist (ABC).

\begin{tabular}{|c|c|c|c|c|c|c|}
\hline Original & R1 & ER & EG & R2 & ER & EG \\
\hline $\begin{array}{l}\text { 1. Excessively active at home, school, } \\
\text { work, or elsewhere }\end{array}$ & $\begin{array}{l}\text { 1. Excessively active at home, } \\
\text { school and in other places }\end{array}$ & 90 & PA & $\begin{array}{l}\text { 1. Excessively active at home, at } \\
\text { school, at work, or any other place }\end{array}$ & 100 & IN \\
\hline 2. Injures self on purpose & 2. Injures himself/herself deliberately & 100 & IN & 2. Intentional self-aggression & 50 & MA \\
\hline 3. Listless, sluggish, inactive & 3. Indifferent, slow, inactive & 100 & IN & 3. Indifferent, lazy, passive & 60 & MA \\
\hline $\begin{array}{l}\text { 4. Aggressive to other children or } \\
\text { adults (verbally or physically) }\end{array}$ & $\begin{array}{l}\text { 4. Aggressive to other children or } \\
\text { adults (verbally or physically) }\end{array}$ & 100 & IN & $\begin{array}{l}\text { 4. Aggressive with other children or } \\
\text { adults (verbally or physically) }\end{array}$ & 100 & IN \\
\hline 5. Seeks isolation from others & 5. Tries to isolate himself from others & 100 & IN & 5. Tries to isolate himself from others & 100 & IN \\
\hline $\begin{array}{l}\text { 6. Meaningless, recurring body } \\
\text { movements }\end{array}$ & $\begin{array}{l}\text { 6. Repetitive, meaningless body } \\
\text { movements }\end{array}$ & 100 & IN & $\begin{array}{l}\text { 6. Constant, meaningless body } \\
\text { movements }\end{array}$ & 80 & PA \\
\hline $\begin{array}{l}\text { 7. Boisterous (inappropriately noisy } \\
\text { and rough) }\end{array}$ & $\begin{array}{l}\text { 7. Noisy (rude and inappropriate } \\
\text { noises) }\end{array}$ & 80 & PA & $\begin{array}{l}\text { 7. Turbulent, inappropriately } \\
\text { noisy and rude }\end{array}$ & 80 & PA \\
\hline 8. Screams inappropriately & 8. Screams inappropriately & 100 & IN & 8. Screams inappropriately & 100 & IN \\
\hline 9. Talks excessively & 9. Talks excessively & 100 & IN & 9. Talks excessively & 100 & IN \\
\hline 10. Temper tantrums/outbursts & 10. Temper tantrums & 100 & IN & 10. Bad-tempered, fits of rage & 50 & MA \\
\hline $\begin{array}{l}\text { 11. Stereotyped behavior; abnormal, } \\
\text { repetitive movements }\end{array}$ & $\begin{array}{l}\text { 11. Stereotypical behavior; } \\
\text { abnormal repetitive movements }\end{array}$ & 100 & IN & $\begin{array}{l}\text { 11. Stereotypical behavior; abnormal, } \\
\text { repeated movements }\end{array}$ & 90 & IN \\
\hline 12. Preoccupied; stares into space & 12. Worried; stares into space & 100 & IN & 12. Worried, head in the clouds & 50 & MA \\
\hline 13. Impulsive (acts without thinking) & 13. Impulsive (acts without thinking) & 100 & IN & 13. Impulsive (acts without thinking) & 100 & IN \\
\hline 14. Irritable and whiny & 14. Irritable and whiny & 100 & IN & 14. Irritable and complaining & 50 & MA \\
\hline 15. Restless, unable to sit still & 15. Restless, unable to sit still & 100 & IN & 15. Restless, incapable of staying still & 100 & IN \\
\hline 16. Withdrawn; prefers solitary activities & $\begin{array}{l}\text { 16. Withdrawn; prefers solitary } \\
\text { activities }\end{array}$ & 100 & IN & 16. Isolation; prefers solitary activities & 80 & PA \\
\hline 17. Odd, bizarre in behavior & 17. Eccentric, odd behavior & 100 & IN & 17. Weird, bizarre behavior & 80 & PA \\
\hline 18. Disobedient; difficult to control & 18. Disobedient; difficult to control & 100 & IN & 18. Disobedient; hard to control & 100 & IN \\
\hline 19. Yells at inappropriate times & 19. Yells at inappropriate moments & 100 & IN & 19. Shouts at inappropriate moments & 100 & IN \\
\hline $\begin{array}{l}\text { 20. Fixed facial expression; lacks } \\
\text { emotional responsiveness }\end{array}$ & $\begin{array}{l}\text { 20. Fixed facial expressions; } \\
\text { lack of emotional response }\end{array}$ & 100 & IN & $\begin{array}{l}\text { 20. Rigid facial expression; } \\
\text { lack of emotion }\end{array}$ & 80 & PA \\
\hline 21. Disturbs others & 21. Annoys other people & 85 & PA & 21. Disturbs others & 100 & IN \\
\hline 22. Repetitive speech & 22. Speaks repetitively & 100 & IN & 22. Repetitive speech & 100 & IN \\
\hline $\begin{array}{l}\text { 23. Does nothing but sit and watch } \\
\text { others }\end{array}$ & $\begin{array}{l}\text { 23. Does nothing but sit and } \\
\text { look at others }\end{array}$ & 100 & IN & $\begin{array}{l}\text { 23. Does nothing but sit and } \\
\text { look at others }\end{array}$ & 100 & IN \\
\hline 24. Uncooperative & 24. Is uncooperative & 100 & IN & 24. Does not cooperate & 100 & IN \\
\hline 25. Depressed mood & 25. Depressed & 80 & PA & 25. Depressive & 100 & IN \\
\hline 26. Resists any form of physical contact & $\begin{array}{l}\text { 26. Resists any form of } \\
\text { physical contact }\end{array}$ & 100 & IN & $\begin{array}{l}\text { 26. Resists any kind of } \\
\text { physical contact }\end{array}$ & 100 & IN \\
\hline $\begin{array}{l}\text { 27. Moves or rolls head back and forth } \\
\text { repetitively }\end{array}$ & $\begin{array}{l}\text { 27. Moves head back and } \\
\text { forth repeatedly }\end{array}$ & 100 & IN & $\begin{array}{l}\text { 27. Moves head to and fro } \\
\text { repetitively }\end{array}$ & 100 & IN \\
\hline $\begin{array}{l}\text { 28. Does not pay attention } \\
\text { to instructions }\end{array}$ & 28. Pays no attention to instructions & 100 & IN & $\begin{array}{l}\text { 28. Does not pay attention } \\
\text { to instructions }\end{array}$ & 100 & IN \\
\hline 29. Demands must be met immediately & $\begin{array}{l}\text { 29. Demands have to be met } \\
\text { immediately }\end{array}$ & 100 & IN & $\begin{array}{l}\text { 29. Requests have to be immediately } \\
\text { satisfied }\end{array}$ & 100 & IN \\
\hline $\begin{array}{l}\text { 30. Isolates himself/herself from other } \\
\text { children or adults }\end{array}$ & $\begin{array}{l}\text { 30. Isolates him/herself from other } \\
\text { children or adults }\end{array}$ & 100 & IN & $\begin{array}{l}\text { 30. Isolates him/herself from other } \\
\text { children or adults }\end{array}$ & 100 & IN \\
\hline 31. Disrupts group activities & 31. Hampers group activities & 100 & IN & 31. Disrupts group activities & 100 & IN \\
\hline
\end{tabular}

(continua) 
Tabela 1 (continuação)

\begin{tabular}{|c|c|c|c|c|c|c|}
\hline Original & R1 & ER & EG & R2 & ER & EG \\
\hline $\begin{array}{l}\text { 32. Sits or stands in one position } \\
\text { for a longtime }\end{array}$ & $\begin{array}{l}\text { 32. Remains seated or standing in } \\
\text { the same position for long periods } \\
\text { of time }\end{array}$ & 100 & IN & $\begin{array}{l}\text { 32. Sits or stands in the same } \\
\text { position for a longtime }\end{array}$ & 100 & IN \\
\hline 33. Talks to self loudly & 33. Talks out loud to him/herself & 100 & IN & 33. Talks out loud to him/herself & 100 & IN \\
\hline $\begin{array}{l}\text { 34. Cries over minor annoyances and } \\
\text { hurts }\end{array}$ & $\begin{array}{l}\text { 34. Cries over minor grievances } \\
\text { or injuries }\end{array}$ & 100 & IN & 34. Cries for minor things & 60 & MA \\
\hline $\begin{array}{l}\text { 35. Repetitive hand, body, } \\
\text { or head movements }\end{array}$ & $\begin{array}{l}\text { 35. Repetitive movements of hands, } \\
\text { head or body }\end{array}$ & 100 & IN & $\begin{array}{l}\text { 35. Repetitive movements of hands, } \\
\text { body or head }\end{array}$ & 100 & IN \\
\hline 36. Mood changes quickly & 36. Rapid mood changes & 100 & IN & 36. Rapid mood changes & 100 & IN \\
\hline $\begin{array}{l}\text { 37. Unresponsive to structured activities } \\
\text { (does not react) }\end{array}$ & $\begin{array}{l}\text { 37. Does not follow structured } \\
\text { activities }\end{array}$ & 80 & PA & $\begin{array}{l}\text { 37. Indifferent to structured activities } \\
\text { (does not react) }\end{array}$ & 100 & IN \\
\hline $\begin{array}{l}\text { 38. Does not stay in seat } \\
\text { (e.g., during lesson or training } \\
\text { periods, meals, etc.) }\end{array}$ & $\begin{array}{l}\text { 38. Will not remain seated } \\
\text { (e.g., during meals, homework or any } \\
\text { training, etc) }\end{array}$ & 100 & IN & $\begin{array}{l}\text { 38. Does not stay seated } \\
\text { (e.g., during classes or training } \\
\text { periods, meals etc) }\end{array}$ & 100 & IN \\
\hline $\begin{array}{l}\text { 39. Will not sit still for any length } \\
\text { of time }\end{array}$ & $\begin{array}{l}\text { 39. Will not remain seated even for } \\
\text { a short time }\end{array}$ & 90 & IN & $\begin{array}{l}\text { 39. Incapable of remaining seated } \\
\text { and quiet }\end{array}$ & 80 & PA \\
\hline $\begin{array}{l}\text { 40. Is difficult to reach, contact or } \\
\text { get through to }\end{array}$ & $\begin{array}{l}\text { 40. It is difficult to reach, } \\
\text { contact or get to him/her }\end{array}$ & 90 & IN & $\begin{array}{l}\text { 40. Hard to catch up with him/her, } \\
\text { contact him/her or approach him/her }\end{array}$ & 50 & MA \\
\hline 41. Cries and screams inappropriately & $\begin{array}{l}\text { 41. Cries and screams } \\
\text { inappropriately }\end{array}$ & 100 & IN & 41. Cries and shouts inappropriately & 100 & IN \\
\hline 42. Prefers to be alone & 42. Prefers to be alone & 100 & IN & 42. Prefers being alone & 100 & IN \\
\hline $\begin{array}{l}\text { 43. Does not try to communicate } \\
\text { by words or gestures }\end{array}$ & $\begin{array}{l}\text { 43. Does not try to communicate } \\
\text { with words or gestures }\end{array}$ & 100 & IN & $\begin{array}{l}\text { 43. Does not try to communicate } \\
\text { with words or gestures }\end{array}$ & 100 & IN \\
\hline 44. Easily distractible & 44. Is easily distracted & 100 & IN & 44. Easily self-distracted & 100 & IN \\
\hline $\begin{array}{l}\text { 45. Waves or shakes the extremities } \\
\text { repeatedly }\end{array}$ & $\begin{array}{l}\text { 45. Shakes his/her extremities } \\
\text { repeatedly }\end{array}$ & 100 & IN & $\begin{array}{l}\text { 45. Shakes and swings extremities } \\
\text { repeatedly }\end{array}$ & 100 & IN \\
\hline $\begin{array}{l}\text { 46. Repeats a word or phrase } \\
\text { over and over }\end{array}$ & $\begin{array}{l}\text { 46. Repeats words or phrases } \\
\text { several times }\end{array}$ & 100 & IN & $\begin{array}{l}\text { 46. Repeats a words or sentence } \\
\text { several times }\end{array}$ & 100 & IN \\
\hline $\begin{array}{l}\text { 47. Stamps feet or bangs objects } \\
\text { or slams doors }\end{array}$ & $\begin{array}{l}\text { 47. Stamps feet, bangs objects } \\
\text { or hits doors hard }\end{array}$ & 100 & IN & $\begin{array}{l}\text { 47. Stamps feet on the ground, or } \\
\text { hits objects or slams doors }\end{array}$ & 100 & IN \\
\hline $\begin{array}{l}\text { 48. Constantly runs or jumps around } \\
\text { the room }\end{array}$ & $\begin{array}{l}\text { 48. Constantly runs or jumps } \\
\text { around the room }\end{array}$ & 100 & IN & $\begin{array}{l}\text { 48. Runs or leaps around the room } \\
\text { constantly }\end{array}$ & 100 & IN \\
\hline $\begin{array}{l}\text { 49. Rocks body back and forth } \\
\text { repeatedly }\end{array}$ & $\begin{array}{l}\text { 49. Rocks his/her body backwards } \\
\text { and forwards repeatedly }\end{array}$ & 100 & IN & $\begin{array}{l}\text { 49. Swings his/her body to and } \\
\text { fro repeatedly }\end{array}$ & 100 & IN \\
\hline 50. Deliberately hurts himself/herself & 50. Deliberately hurts him/herself & 100 & IN & 50. Harms him/herself deliberately & 60 & MA \\
\hline $\begin{array}{l}\text { 51. Pays no attention when } \\
\text { spoken to }\end{array}$ & $\begin{array}{l}\text { 51. Pays no attention when } \\
\text { spoken to }\end{array}$ & 100 & IN & $\begin{array}{l}\text { 51. Does not pay attention when } \\
\text { someone speaks to him/her }\end{array}$ & 100 & IN \\
\hline 52. Does physical violence to self & 52. Is violent against him/herself & 100 & IN & $\begin{array}{l}\text { 52. Is physically aggressive to } \\
\text { him/herself }\end{array}$ & 100 & IN \\
\hline $\begin{array}{l}\text { 53. Inactive, never moves } \\
\text { spontaneously }\end{array}$ & $\begin{array}{l}\text { 53. Inactive, never moves } \\
\text { spontaneously }\end{array}$ & 100 & IN & $\begin{array}{l}\text { 53. Inactive, never moves } \\
\text { spontaneously }\end{array}$ & 100 & IN \\
\hline 54. Tends to be excessively active & 54. Tends to be excessively active & 100 & IN & 54. Tends to be hyperactive & 80 & MA \\
\hline 55. Responds negatively to affection & 55. Responds negatively to affection & 100 & IN & 55. Responds negatively to affection & 100 & IN \\
\hline 56. Deliberately ignores directions & 56. Deliberately ignores direction & 100 & IN & 56. Ignores instructions deliberately & 100 & IN \\
\hline $\begin{array}{l}\text { 57. Has temper outbursts or tantrums } \\
\text { when he/she does not get own way }\end{array}$ & $\begin{array}{l}\text { 57. Has a temper tantrum when he/ } \\
\text { she does not get his/her own way }\end{array}$ & 100 & IN & $\begin{array}{c}\text { 57. Has fits of rage or bad temper } \\
\text { when he/she does not get things } \\
\text { done his/her own way }\end{array}$ & 100 & IN \\
\hline $\begin{array}{l}\text { 58. Shows few social reactions } \\
\text { to others }\end{array}$ & $\begin{array}{l}\text { 58. Shows little social reactions } \\
\text { to others }\end{array}$ & 100 & IN & $\begin{array}{l}\text { 58. Shows poor social reactions with } \\
\text { respect to others }\end{array}$ & 100 & IN \\
\hline
\end{tabular}

CA: completamente alterado; EG: avaliação da equivalência geral; ER: avaliação da equivalência referencial (porcentagem de semelhança); IN: inalterado; MA: muito alterado; PA: pouco alterado; R1: primeira "retrotradução"; R2: segunda "retrotradução". 
Versão-síntese submetida ao pré-teste, com itens discriminados segundo a origem (T1 ou T2).

\begin{tabular}{|c|c|c|c|}
\hline Item & & Item & \\
\hline $\begin{array}{l}\text { 1. Excessivamente ativo(a) em casa, } \\
\text { na escola, no trabalho ou em qualquer lugar }\end{array}$ & Modificada de T2 & 30. Isola-se de outras crianças ou de adultos & $\mathrm{T} 1$ \\
\hline 2. Fere-se de propósito & Modificada de T1 & 31. Tumultua as atividades em grupo & Modificada \\
\hline 3. Indiferente, lento(a), parado(a) & Modificada de T1 & $\begin{array}{l}\text { 32. Fica sentado(a) ou em pé na mesma posição } \\
\text { por muito tempo }\end{array}$ & $\mathrm{T} 1$ \\
\hline $\begin{array}{l}\text { 4. Agressivo(a) com outras crianças ou adultos } \\
\text { (verbalmente ou fisicamente) }\end{array}$ & $\mathrm{T} 2$ & 33. Fala sozinho(a) em voz alta & $\mathrm{T} 2$ \\
\hline 5. Procura se isolar dos outros & Modificada de T2 & $\begin{array}{l}\text { 34. Chora por mínimos aborrecimentos } \\
\text { e machucados }\end{array}$ & Modificada de T1 \\
\hline $\begin{array}{l}\text { 6. Movimentos corporais repetitivos } \\
\text { e sem sentido }\end{array}$ & Modificada de T1 & $\begin{array}{l}\text { 35. Movimentos repetitivos das mãos, } \\
\text { do corpo ou da cabeça }\end{array}$ & $\mathrm{T} 2$ \\
\hline $\begin{array}{l}\text { 7. Barulhento(a) (ruídos grosseiros e } \\
\text { inapropriados) }\end{array}$ & $\mathrm{T} 1$ & 36. O humor muda rapidamente & T1 \\
\hline 8. Grita inapropriadamente & $\mathrm{T} 1, \mathrm{~T} 2$ & $\begin{array}{l}\text { 37. Indiferente às atividades estruturadas } \\
\text { (não reage) }\end{array}$ & $\mathrm{T} 2$ \\
\hline 9. Fala excessivamente & $\mathrm{T} 1, \mathrm{~T} 2$ & $\begin{array}{l}\text { 38. Não permanece sentado (ex.: durante as } \\
\text { lições ou outras atividades, refeições etc.) }\end{array}$ & Modificada de T1 \\
\hline 10. Crises de birra/acesso de fúria & Modificada de T1 & $\begin{array}{l}\text { 39. Não fica sentado(a) nem por } \\
\text { um tempo mínimo }\end{array}$ & $\mathrm{T} 1$ \\
\hline $\begin{array}{l}\text { 11. Comportamentos estereotipados; } \\
\text { movimentos anormais, repetitivos }\end{array}$ & $\mathrm{T} 1$ & $\begin{array}{l}\text { 40. Difícil alcançá-lo(a), contatá-lo(a) ou } \\
\text { chegar até ele(a) }\end{array}$ & $\mathrm{T} 1, \mathrm{~T} 2$ \\
\hline $\begin{array}{l}\text { 12. Preocupado(a), absorto(a), distraído(a); } \\
\text { fixa o olhar no vazio }\end{array}$ & Modificada de T1 & 41. Chora e grita inapropriadamente & $\mathrm{T} 2$ \\
\hline 13. Impulsivo(a) (age sem pensar) & $\mathrm{T} 1, \mathrm{~T} 2$ & 42. Prefere ficar sozinho(a) & $\mathrm{T} 1, \mathrm{~T} 2$ \\
\hline 14. Irritável e queixoso(a) & T1 & $\begin{array}{l}\text { 43. Não tenta se comunicar por palavras } \\
\text { ou gestos }\end{array}$ & $\mathrm{T} 1, \mathrm{~T} 2$ \\
\hline $\begin{array}{l}\text { 15. Inquieto(a), incapaz de permanecer } \\
\text { sentado(a) }\end{array}$ & $\mathrm{T} 1$ & 44. Distrai-se com facilidade & T1 \\
\hline 16. Retraído(a); prefere atividades solitárias. & $\mathrm{T} 1$ & $\begin{array}{l}\text { 45. Balança ou agita as extremidades } \\
\text { repetidamente }\end{array}$ & Modificada de T1 \\
\hline 17. Excêntrico(a), comportamento bizarro & Modificada de T1 & 46. Repete várias vezes uma palavra ou frase & $\mathrm{T} 2$ \\
\hline 18. Desobediente; difícil de controlar & $\mathrm{T} 1, \mathrm{~T} 2$ & $\begin{array}{l}\text { 47. Bate os pés, ou faz barulho estrondoso com } \\
\text { objetos ou bate portas com força }\end{array}$ & Modificada de T1 \\
\hline 19. Grita em momentos inapropriados & $\mathrm{T} 1$ & $\begin{array}{l}\text { 48. Constantemente corre ou pula em torno } \\
\text { do cômodo }\end{array}$ & T1 \\
\hline $\begin{array}{l}\text { 20. Expressão facial imóvel, fixa; } \\
\text { falta de resposta emocional }\end{array}$ & Modificada de T1 & $\begin{array}{c}\text { 49. Balança o corpo para trás e para frente } \\
\text { repetidamente }\end{array}$ & $\mathrm{T} 2$ \\
\hline 21. Incomoda os outros & $\mathrm{T} 2$ & 50. Causa machucados em si mesmo & Modificada de T1 \\
\hline 22. Fala repetitiva & $\mathrm{T} 1$ & 51. Não presta atenção quando falam com ele(a) & $\mathrm{T} 1, \mathrm{~T} 2$ \\
\hline $\begin{array}{l}\text { 23. Não faz nada a não ser ficar sentado(a) e } \\
\text { olhar os outros }\end{array}$ & $\mathrm{T} 1, \mathrm{~T} 2$ & 52. Pratica violência contra si próprio & $\mathrm{T} 1$ \\
\hline 24. Não é cooperativo & $\mathrm{T} 1$ & 53. Inativo(a), nunca se move espontaneamente & $\mathrm{T} 2$ \\
\hline 25. Depressivo(a) & $\mathrm{T} 2$ & 54. Tende a ser excessivamente ativo(a) & Modificada de T1 \\
\hline 26. Resiste a qualquer forma de contato físico & $\mathrm{T} 1, \mathrm{~T} 2$ & 55. Reage negativamente ao contato afetivo & Modificada de T1 \\
\hline $\begin{array}{l}\text { 27. Movimenta ou balança a cabeça de trás } \\
\text { para frente repetidamente }\end{array}$ & $\mathrm{T} 1$ & 56. Ignora deliberadamente as instruções & $\mathrm{T} 2$ \\
\hline 28. Não presta atenção às instruções & $\mathrm{T} 1, \mathrm{~T} 2$ & $\begin{array}{l}\text { 57. Tem acesso de fúria ou birra quando } \\
\text { contrariado }\end{array}$ & Modificada \\
\hline $\begin{array}{l}\text { 29. Os pedidos têm que ser atendidos } \\
\text { imediatamente }\end{array}$ & $\mathrm{T} 1$ & 58. Demonstra pouca reação social aos outros & $\mathrm{T} 2$ \\
\hline
\end{tabular}


Modificações realizadas após aplicação do pré-teste.

\begin{tabular}{lcc}
\hline Item & Antes & Depois \\
\hline 12 & Preocupado(a), absorto(a), distraído(a); fixa o olhar no vazio & Preocupado(a), distraído(a), fixa o olhar no vazio \\
17 & Excêntrico, comportamento bizarro & Estranho, comportamento esquisito \\
37 & Indiferente às atividades estruturadas (não reage) & Balança ou agita mãos ou pés repetidamente \\
45 & Balança ou agita as extremidades repetidamente & Ignora propositalmente as instruções \\
56 & Ignora deliberadamente as instruções &
\end{tabular}

No item 56, que apresentou problemas de compreensão apenas na amostra de familiares, a palavra "deliberadamente" foi substituída por "propositalmente", sinônimo mais corriqueiro, o que pode torná-la mais acessível a pessoas com graus de escolaridade mais baixos.

A expressão "comportamentos estereotipados”, presente no item 11, é utilizada em semiologia mental, e não gerou problemas na amostra de professores. Optou-se por mantê-la, uma vez que vem acompanhada pelo aposto "movimentos anormais, repetitivos", compreendido pela totalidade da amostra.

Da mesma forma, decidiu-se manter o item 20 ("expressão facial imóvel, fixa, falta de resposta emocional") inalterado, uma vez que gerou problemas para uma minoria da amostra de familiares (15\%) e descreve comportamento evidente em determinadas doenças mentais (autismo, por exemplo), acreditando-se que alguma familiaridade com as mesmas é suficiente para a sua compreensão.

\section{Discussão}

O retardo mental é um transtorno psiquiátrico com alta prevalência e muito associado à comorbidades, o que muitas vezes torna necessário um tratamento, seja ele farmacológico ou psicopedagógico. Tendo em vista a dificuldade de mensurar a resposta terapêutica, a escala $\mathrm{ABC}$ propõe-se a avaliar esse importante parâmetro na clínica, principalmente psiquiátrica.

Uma das formas de se avaliar inteligência é a desenvolvida por David Wechsler, que em 1949, elaborou as Escalas Wechsler de Inteligência. Estas categorizam os resultados em forma de QI, verbal ou não verbal, ou de execução. A partir de então, o QI passou a ser a forma mais utilizada de quantificar inteligência. A escala $\mathrm{ABC}$ não avalia QI, porque não testa inteligência. Seu objetivo é verificar melhora de sintomas psiquiátricos em portadores de retardo mental, e por isso, esse estudo traz uma grande contribuição para a prática clínica.

Outra grande diferença entre a $\mathrm{ABC}$ e as demais escalas é que aquela pode ser aplicada por qualquer pessoa que tenha contato com o indívíduo a ser analisado, seja profissional da área de saúde, familiar ou professor. Já as Escalas Wechsler de Inteligência, que estão entre as mais usadas para avaliar inteligência, exigem que seu aplicador seja altamente treinado tanto para a aplicação quanto para a correção. Esta diferença garante a utilização da $\mathrm{ABC}$ na prática cotidiana, já que as outras escalas têm na sua formulação testes técnicos, como Raciocínio Matricial (medida do processamento da informação visual e raciocínio abstrato), Sequência de Números e Letras, dentre outros.

Em psiquiatria, a linguagem é o principal meio de avaliação do paciente. Tanto a forma verbal como a não verbal orientam o psiquiatra quanto aos sintomas. O conteúdo do discurso, principalmente, é o que mais auxilia no exame psíquico. Dessa forma, fica evidente a importância de se realizar uma tradução pormenorizada quando se trata de instrumentos que serão usados para avaliar sintomas psiquiátricos. Este estudo propõe-se a realizar a tradução em etapas para ser o mais fidedigno possível com o original, mas adaptando-se à linguagem de destino, o português do Brasil.

O estudo foi realizado seguindo as etapas de avaliação conceitual e de itens, tradução, retradução, avaliação dos significados denotativo e conotativo e aplicação do pré-teste. Foram omitidas as etapas de equivalência de mensuração e funcional, que serão realizadas num estudo próximo, para a conclusão de uma efetiva adaptação transcultural.

A quinta etapa da equivalência semântica, o pré-teste, evidenciou uma boa compreensão da escala por parte dos professores de escolas especializadas, uma vez que $94,8 \%$ dos itens foram 
compreendidos pela integralidade da amostra. As dúvidas se concentraram em torno de três itens, 12, 17 e 45, do universo de 58 itens componentes da escala.

A amostra de familiares de portadores de retardo teve uma compreensão razoável da escala, sendo que $89,6 \%$ dos itens foram compreendidos pela totalidade da amostra. Mais uma vez, os itens 12, 17 e 45 geraram dúvidas quanto ao vocabulário empregado, e na amostra de familiares, os itens 11, 20, 37 e 56 também se mostraram problemáticos.

Atribuiu-se a diferença no grau de compreensão entre as amostras sobretudo à diferença de escolaridade. Enquanto que a amostra de professores era composta por $90 \%$ de indivíduos que haviam concluído o ensino superior, e ainda contava com $25 \%$ dos integrantes em cursos de pósgraduação, apenas $20 \%$ da amostra de pais possuíam ensino superior completo, sendo que $45 \%$ da amostra não haviam concluído nem o ensino médio. Além disso, observou-se que alguns itens apresentavam um jargão técnico com o qual a amostra de professores tinha mais probabilidade de estar familiarizada. Dessa forma, optou-se por modificar a maioria dos itens geradores de dúvidas, de modo a tornar a escala mais compreensível para a população-alvo (Tabela 3).

Os autores deste estudo optaram pela metodologia descrita por Reichenheim \& Moraes 27, dentre as diversas disponíveis na literatura cien- tífica, pois consideraram de importância crucial a análise pormenorizada da avaliação conceitual e de itens e semântica; porém os métodos aqui empregados não cumpriram à risca os passos descritos.

Sugere-se que, como forma de dirimir problemas semânticos, faça-se treinamento prévio dos futuros avaliadores para o esclarecimento das instruções, para a utilização da $\mathrm{ABC}$ em estudos experimentais. Dessa forma, aumenta-se também a sua reprodutibilidade.

\section{Conclusão}

Conforme proposto na introdução, foi realizada a tradução, acrescida da adaptação transcultural parcial da escala $\mathrm{ABC}$, sendo esta considerada adequada por profissionais das áreas de psiquiatria e neurologia, além de compreensível pelos familiares e professores de pacientes portadores de retardo mental. Entretanto, sugere-se que, como forma de dirimir problemas semânticos, faça-se treinamento prévio dos futuros avaliadores para o esclarecimento das instruções, bem como da terminologia, para a utilização da $\mathrm{ABC}$ em estudos experimentais. Fica então disponível na literatura nacional a versão para o português do Brasil da escala ABC (Figura 1), respeitando os aspectos de equivalência conceitual e de itens e semântica. 
Figura 1

Versão final traduzida da Aberrant Behavior Checklist.

Escala de Comportamento Atípico

(ABC - Aberrant Behavior Checklist)

Nome do paciente:

Sexo (circular): Masculino / Feminino

Data de nascimento:

$$
\text { Dia Mês Ano }
$$

Data de hoje:

$$
\text { Dia Mês Ano }
$$

Nome do avaliador:

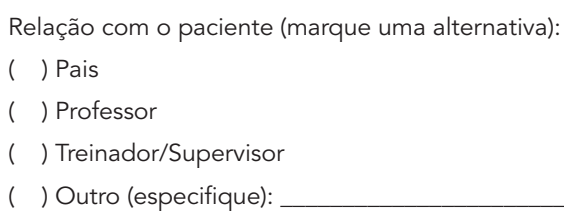

Onde o paciente foi observado:
( ) Casa
( ) Escola
( ) Creche
( ) Consultório
( ) Outro (especifique):

Caso esteja na escola, qual o tipo (marque uma alternativa):

( ) Escola para portadores de necessidades especiais.

( ) Escola para portadores de autismo.

( ) Escola de inclusão

( ) Escola comum

( ) Outros (especifique):

Grupo étnico (marcar):
( ) Pardo
( ) Outros (especifique):
( ) Branco

Situação clínica do paciente (por favor, circule):
a. Surdez?
Não
Sim
? (Não sei)
b. Cegueira?
Não
Sim
? (Não sei)
c. Epilepsia?
Não
Sim
? (Não sei)
Não
Sim
? (Não sei)

e. Outro

MEDICAÇÕES EM USO ATUALMENTE (Por favor, liste todas as medicações e sua dosagem)

1.

2.

3

Nota: (c) 1994 Slosson Educational Publication, Inc.

Cópias adicionais disponíveis em: Slosson Educational Publications, Inc. P.O. Box 28, East Aurora, New York 14052, USA. 


\section{INSTRUÇÕES}

A escala de sintomas $A B C$ comunitária foi elaborada para ser usada em pacientes que vivem em comunidade. Por isso o termo paciente é usado para se referir à pessoa que está sendo avaliada, que pode ser uma criança em idade escolar, um adolescente ou um adulto.

Pontue o comportamento do paciente em relação às quatro últimas semanas. Para cada item decida se o comportamento é um problema e circule o número apropriado:

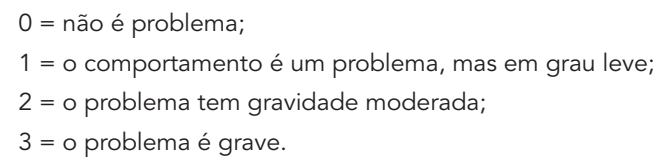

\section{Quando estiver julgando o comportamento do paciente, tenha em mente os pontos a seguir:}

a) Considere a frequência com que cada comportamento acontece de forma relativa. Por exemplo, se um paciente tem em média mais acessos de fúria do que a maioria de outros pacientes que você conhece, ou do que a maioria dos seus colegas de classe, a gravidade é provavelmente moderada (2) ou grave (3), mesmo que ocorra somente uma ou duas vezes por semana. Outros comportamentos, como desobediência, provavelmente precisam ocorrer com maior frequência para merecer a pontuação máxima.

b) Considere a opinião de outros cuidadores do paciente, caso você tenha acesso a essa informação. Se o paciente tem problemas com outros, mas não com você, tente levar em conta a situação de maneira geral.

c) Tente considerar se um determinado comportamento interfere no desenvolvimento, funcionamento ou relacionamento dele/dela. Por exemplo, balançar o corpo ou retraimento social podem não perturbar outras crianças ou adultos, mas certamente atrapalha o funcionamento ou desenvolvimento individual.

Não se detenha muito tempo em cada item, sua primeira impressão geralmente é a correta.

\begin{tabular}{|llllll|}
\hline 1. & Excessivamente ativo(a) em casa, na escola, no trabalho ou em qualquer lugar & 0 & 1 & 2 & 3 \\
2. & Fere-se de propósito & 0 & 1 & 2 & 3 \\
3. & Indiferente, lento(a), parado(a) & 0 & 1 & 2 & 3 \\
4. & Agressivo(a) com outras crianças ou adultos (verbalmente ou fisicamente) & 0 & 1 & 2 & 3 \\
5. & Procura se isolar dos outros & 0 & 1 & 2 & 3 \\
6. & Movimentos corporais repetitivos e sem sentido & 0 & 1 & 2 & 3 \\
7. & Barulhento(a) (ruídos grosseiros e inapropriados) & 0 & 1 & 2 & 3 \\
8. & Grita inapropriadamente & 0 & 1 & 2 & 3 \\
9. & Fala excessivamente & 0 & 1 & 2 & 3 \\
10. & Crises de birra/acesso de fúria & 0 & 1 & 2 & 3 \\
11. & Comportamentos estereotipados; movimentos anormais, repetitivos & 0 & 1 & 2 & 3 \\
12. & Preocupado(a), fixa o olhar no vazio & 0 & 1 & 2 & 3 \\
13. Impulsivo(a) (age sem pensar) & 0 & 1 & 2 & 3 \\
14. Irritável e queixoso(a) & 0 & 1 & 2 & 3 \\
15. Inquieto(a), incapaz de permanecer sentado(a) & 0 & 1 & 2 & 3 \\
16. & Retraído(a); prefere atividades solitárias & 0 & 1 & 2 & 3 \\
17. & Estranho, comportamento esquisito & 0 & 1 & 2 & 3 \\
18. & Desobediente; difícil de controlar & 0 & 1 & 2 & 3 \\
19. & Grita em momentos inapropriados & 0 & 1 & 2 & 3 \\
20. & Expressão facial imóvel, fixa; falta de resposta emocional & 0 & 1 & 2 & 3 \\
\hline
\end{tabular}




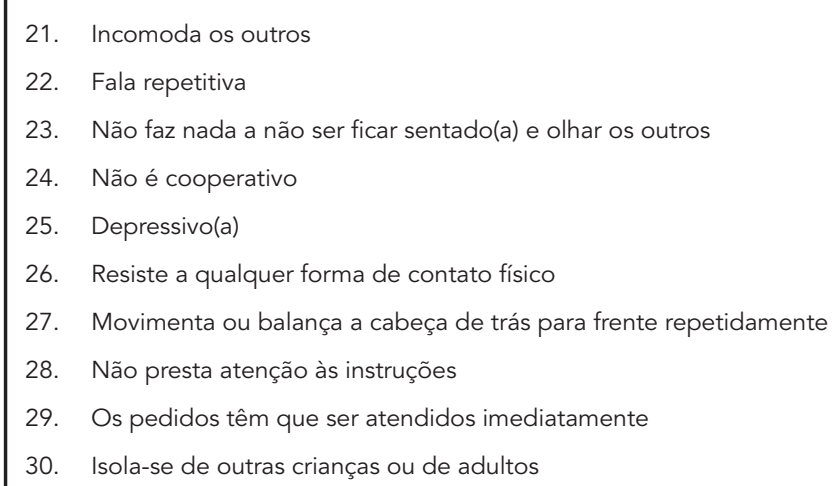

$\begin{array}{llll}0 & 1 & 2 & 3 \\ 0 & 1 & 2 & 3 \\ 0 & 1 & 2 & 3 \\ 0 & 1 & 2 & 3 \\ 0 & 1 & 2 & 3 \\ 0 & 1 & 2 & 3 \\ 0 & 1 & 2 & 3 \\ 0 & 1 & 2 & 3 \\ 0 & 1 & 2 & 3 \\ 0 & 1 & 2 & 3\end{array}$

31. Tumultua as atividades em grupo

32. Fica sentado(a) ou em pé na mesma posição por muito tempo

33. Fala sozinho(a) em voz alta

34. Chora por mínimos aborrecimentos e machucados

35. Movimentos repetitivos das mãos, do corpo ou da cabeça

36. O humor muda rapidamente

37. Não acompanha as atividades estruturadas (não reage)

38. Não permanece sentado (ex. durante as lições ou outras atividades, refeições etc.)

39. Não fica sentado(a) nem por um tempo mínimo

40. Difícil alcançá-lo(la), contatá-lo(la) ou chegar até ele(ela)

41. Chora e grita inapropriadamente

42. Prefere ficar sozinho(a)

43. Não tenta se comunicar por palavras ou gestos

44. Distrai-se com facilidade

45. Balança ou agita as mãos ou pés repetidamente

46. Repete várias vezes uma palavra ou frase

47. Bate os pés, ou faz barulho estrondoso com objetos ou bate portas com força

48. Constantemente corre ou pula em torno do cômodo

49. Balança o corpo para trás e para frente repetidamente

50. Causa machucados em si mesmo

$\begin{array}{llll}0 & 1 & 2 & 3 \\ 0 & 1 & 2 & 3 \\ 0 & 1 & 2 & 3 \\ 0 & 1 & 2 & 3 \\ 0 & 1 & 2 & 3 \\ 0 & 1 & 2 & 3 \\ 0 & 1 & 2 & 3 \\ 0 & 1 & 2 & 3 \\ 0 & 1 & 2 & 3 \\ 0 & 1 & 2 & 3\end{array}$

\begin{tabular}{|llllll} 
51. & Não presta atenção quando falam com ele(ela) & 0 & 1 & 2 & 3 \\
52. & Pratica violência contra si próprio & 0 & 1 & 2 & 3 \\
53. Inativo(a), nunca se move espontaneamente & 0 & 1 & 2 & 3 \\
54. & Tende a ser excessivamente ativo(a) & 0 & 1 & 2 & 3 \\
55. & Reage negativamente ao contato afetivo & 0 & 1 & 2 & 3 \\
56. & Ignora propositalmente as instruções & 0 & 1 & 2 & 3 \\
57. & Tem acesso de fúria ou birra quando contrariado & 0 & 1 & 2 & 3 \\
58. & Demonstra pouca reação social aos outros & 0 & 1 & 2 & 3
\end{tabular}

Nota: tradução de Mirella Fiuza Losapio, Lis Gomes Silva e Milena Pereira Pondé. 


\section{Resumo}

A ABC (Aberrant Behavior Checklist) visa avaliar a resposta ao tratamento de transtornos comportamentais em portadores de retardo mental. O objetivo deste estudo é descrever a adaptação transcultural parcial da escala ABC para o português do Brasil. Foi realizada avaliação da equivalência conceitual e de itens, foram feitas duas traduções (T1 e T2), suas respectivas retraduções (R1 e R2), avaliação das equivalências referencial e geral, avaliação de especialistas, pré-teste e elaboração da versão final. Em relação à equivalência conceitual e de itens a $A B C$ foi considerada pertinente à nossa cultura. Quanto à equivalência semântica, houve uma boa correspondência entre os itens de R1 e a ABC original, e razoável entre estes e R2. Portanto optou-se por utilizar os itens de T1. Todos os professores compreenderam $94,8 \%$ da escala, enquanto todos os parentes entenderam 87,9\%. Fica disponivel a versão em português do Brasil da escala $A B C$, respeitando a equivalência conceitual e de itens e semântica.

Tradução (Processo); Retardo Mental; Resultado de Tratamento; Questionários

\section{Colaboradores}

M. F. Losapio, L. G. Silva e M. P. Pondé redigiram o artigo. Todas as autoras revisaram e aprovaram a versão final do artigo.

\section{Agradecimentos}

À Fundação de Amparo à Pesquisa do Estado da Bahia.

\section{Referências}

1. Kabra M, Gulati S. Mental retardation. Indian J Pediatr 2003; 70:153-8.

2. Accardo PJ, Capute AJ. Mental retardation. In: Capute AJ, Accardo PJ, editors. Developmental disabilities in infancy and childhood. Baltimore: Paul H. Brookes Publishing Co.; 1996. p. 525-47.

3. Aicardi J. Mental retardation in diseases of the nervous system in childhood. New York: Cambridge University Press; 1998.

4. Vasconcelos MM. Retardo mental. J Pediatr (Rio J.) 2004; 80 Suppl 2:71-82.

5. Brown EC, Aman MG, Lecavalier L. Empirical classification of behavioral and psychiatric problems in children and adolescents with mental retardation. Am J Ment Retard 2004; 109:445-55.

6. Sundheim STPV, Ryan RM, Voeller KKS. Mental retardation. In: Coffey CE, Brumback RA, editors. Textbook of pediatric neuropsychiatry. Washington DC: American Psychiatric Press; 1998; p. 649-90.

7. Cans C, Wilhelm L, Baile MF, du Mazaubrun C, Grandjean H, Rumeau-Rouquette C. Aetiological findings and associated factors in children with severe mental retardation. Dev Med Child Neurol 1999; 41:233-9.
8. Blacher J, McIntyre LL. Syndrome specificity and behavioural disorders in young adults with intellectual disability: cultural differences in family impact. J Intellect Disabil Res 2006; 50:184-98.

9. Suresh P, Sebastian S. Developmental Gerstmann's syndrome: a distinct clinical entity of learning disabilities. Pediatric Neurol 2000; 22:267-78.

10. Figueiredo VLM. Uma adaptação brasileira do teste de inteligência WISC-III [Tese de Doutorado]. Brasília: Curso de Pós-graduação em Psicologia, Universidade de Brasília; 2001.

11. Nascimento E. Adaptação e validação do teste WAIS-III para um contexto brasileiro [Tese de Doutorado]. Brasília: Curso de Pós-graduação em Psicologia, Universidade de Brasília; 2000.

12. Wechsler SM, Schelini P.W. Bateria de Habilidades Cognitivas Woodcock-Johnson III: validade de construto. Psicol Teor Pesqui 2006; 22:287-96.

13. Raven JC. Matrizes progressivas: escala geral. Rio de Janeiro. Centro de Psicologia Aplicada; 2000.

14. Boccalandro ER. G-36: teste não verbal de inteligência. São Paulo: Vetor; 2002.

15. Wechsler SM. O desenho da figura humana. Campinas: Editora Psy; 2000. 
16. Aman MG, Singh NN, Stewart AW, Field CJ. The Aberrant Behavior Checklist: a behavior rating scale for the assessment of treatment effects. Am J Ment Defic 1985; 89:485-91.

17. Aman MG, Singh NN, Turbott SH. Reliability of the Aberrant Behavior Checklist and the effect of variations in instructions. Am J Ment Defic 1987; 92:237-40.

18. Aman MG, Singh NN, Stewart AW, Field CJ. Psychometric characteristics of the Aberrant Behavior Checklist. Am J Ment Defic 1985; 89:492-502.

19. Freund LS, Reiss AL. Rating problem behaviors in outpatients with mental retardation: use of the Aberrant Behavior Checklist. Res Dev Disabil 1991; 12:435-51.

20. Rojahn J, Helsel WJ. The Aberrant Behavior Checklist in children and adolescents with dual diagnosis. J Autism Dev Disord 1991; 21:17-28.

21. Newton JT, Sturmey P. The Aberrant Behaviour Checklist: a British replication and extension of its psychometric properties. J Ment Defic Res 1988; 32:87-92.

22. Aman MG, Richmond G, Stewart AW, Bell JC, Kissel RC. The Aberrant Behavior Checklist: factor structure and the effect of subject variables in American and New Zealand facilities. Am J Ment Defic 1987; 91:570-8.

23. Bihm EM, Poindexter AR. Cross-validation of the factor structure of the Aberrant Behavior Checklist for persons with mental retardation. Am J Ment Retard 1991; 96:209-11.
24. Marshburn EC, Aman MG. Factor validity and norms for the Aberrant Behavior Chacklist in a community sample of children with mental retardation. J Autism Dev Disord 1992; 22:357-73.

25. Herdman M, Fox-Rushby J, Badia X. A model of equivalence in the cultural adaptation of HRQoL instruments: the universalist approach. Qual Life Res 1998; 7:323-35.

26. Jorge MR. Adaptação transcultural de instrumentos de pesquisa em saúde mental. Rev Psiquiatr Clin (São Paulo) 1998; 25:233-9.

27. Reichenheim ME, Moraes CL. Operacionalização de adaptação transcultural de instrumentos de aferição usados em epidemiologia. Rev Saúde Pública 2007; 41:665-73.

28. Ordudari M. Translation procedures, strategies and methods. Translation Journal 2007; (11). http:// translationjournal.net/journal/41culture.htm.

29. Reichenheim ME, Paixão Jr. CM, Moraes CL. Adaptação transcultural para o português (Brasil) do instrumento Hwalek-Sengstock Elder Abuse Screening Test (H-S/EAST) utilizado para identificar risco de violência contra o idoso. Cad Saúde Pública 2008; 24:1801-13.

30. Streiner DL, Norman GR. Health measurement scales: a practical guide to their development and use. Oxford: Oxford University Press; 2003.

Recebido em 17/Mai/2010

Versão final reapresentada em 02/Fev/2011 Aprovado em 11/Mar/2011 